\title{
Risk of radiation-induced pneumonitis after helical and static-port tomotherapy in lung cancer patients and experimental rats
}

\author{
Xianglan Zhang ${ }^{1,2}$, You Keun Shin ${ }^{3}$, Zhenlong Zheng ${ }^{4}$, Lianhua Zhu ${ }^{4}$ and Ik Jae Lee ${ }^{5^{*}}$
}

\begin{abstract}
Background: Radiotherapy (RT) is one of the major non-operative treatment modalities for treating lung cancer. Tomotherapy is an advanced type of intensity-modulated radiotherapy (IMRT) in which radiation may be delivered in a helical fashion. However, unexpected pneumonitis may occur in patients treated with tomotherapy, especially in combination with chemotherapy, as a result of extensive low-dose radiation of large lung volumes. The aim of our study was to investigate the risk of radiation-induced pneumonitis after helical-mode and static-mode tomotherapy in patients with lung cancer and in an animal model.
\end{abstract}

Method: A total of 63 patients with primary lung cancer who were treated with static or helical tomotherapy with or without concurrent chemoradiotherapy (CCRT) were analyzed. Additionally, rats with radiation-induced pulmonary toxicity, which was induced by the application of helical or static tomography with or without CCRT, were evaluated.

Results: Helical-mode tomotherapy resulted in a significantly higher rate of late radiation pneumonitis in lung cancer patients than static-mode tomotherapy when evaluated by the Radiation Therapy Oncology Group (RTOG) and National Cancer Institute Common Terminology Criteria for Adverse Events (CTCAE) scoring system. In the animal model, helical tomotherapy alone induced significantly higher expression of interleukin (IL)-1a, IL-1 $\beta, I L-6$, and transforming growth factor (TGF)- $\beta$ in lung specimens, especially on the untreated side, compared to static tomotherapy alone. Additionally, rats treated with helical tomotherapy and CCRT demonstrated significantly higher expression of inflammatory cytokines compared to those treated with static tomotherapy and CCRT.

Conclusion: Rat models treated with tomotherapy with or without CCRT could present similar patterns of pulmonary toxicity to those shown in lung cancer patients. The models can be used in further investigations of radiation induced pulmonary toxicity.

\section{Background}

Radiotherapy (RT) is one of the major non-operative treatment modalities used for treating carcinomas of various organs, demonstrated by the fact that approximately half of all cancer patients have been shown to receive RT during their course of anti-cancer treatment $[1,2]$. Ongoing investigations have improved the survival of cancer patients and minimized RT-related toxicities through the design of new RT modalities, including 3-dimensional conformal RT based on computed radiography imaging,

\footnotetext{
* Correspondence: ikjae412@yuhs.ac

${ }^{5}$ Department of Radiation Oncology, Gangnam Severance Hospital, Yonsei University College of Medicine, 211 Eonju-ro, Gangnam-gu, Seoul 135-720, South Korea

Full list of author information is available at the end of the article
}

image-guided RT, stereotactic body RT, and intensitymodulated (IM) RT [2]. Among these modalities, IMRT was developed to deliver irregularly shaped radiation doses as well as to save critical organs through the application of inverse planning software and computercontrolled intensity-modulation (2).

Tomotherapy (Accuray, Palo Alto, USA) is a form of IMRT which is integrated with an image guidance system, and helical tomotherapy delivers IMRT in a continuous helix for improved dose conformity to malignant tissues $[3,4]$. Several oncologic diseases have been effectively and safely treated with helical tomotherapy, including prostate cancer, scalp angiosarcoma, hepatocellular carcinoma, breast cancer, and lung cancer [4-9]. Helical tomotherapy allows delivery of the radiation beam from any gantry 
angle as a result of continuous rotation around the patient, which results in the sparing of surrounding normal tissues from unwanted excessive radiation [3]. Generally, an increased fraction number has been considered to reduce the risk of radiation-induced high-grade toxicities [9]. However, unexpected pneumonitis may occur in patients treated with helical tomotherapy, especially in combination with chemotherapy, as a result of extensive low-dose radiation of large lung volumes $[9,10]$.

To avoid this risk of radiation-induced pneumonitis, a type of static tomotherapy using static gantry positions has been introduced, and a dosimetric study demonstrated optimaltarget volume coverage with adequate sparing of normal tissue with static tomotherapy [11]. In the current study, we compared the risk of radiation pneumonitis in patients with lung cancer who received either helical tomotherapy or static tomotherapy. In addition, we developed rat models of radiation pneumonitis, which were induced by helical tomography and static tomography with or without concurrent chemoradiotherapy (CCRT), respectively. Then, we evaluated the expression of radiation pneumonitis-associated cytokines, including transforming growth factor (TGF)- $\beta$, interleukin (IL)- $1 \alpha$, IL- $1 \beta$, and IL- 6 , to determine the effects of radiation on surrounding normal lung tissues in each animal group.

\section{Materials and methods}

\section{Patients}

This study included 63 patients (43 males and 20 females; median age, 66 years; age range, 33-87 years) diagnosed with primary lung cancer who were treated with helical tomotherapy or static tomotherapy. Baseline characteristics of the participants are summarized in Table 1. The patients were pathologically diagnosed by surgical lung

Table 1 Patients' baseline demographics and characteristics

\begin{tabular}{|c|c|c|}
\hline & & No. of patients (\%) \\
\hline \multirow[t]{2}{*}{ Sex } & Male & $43(68.3)$ \\
\hline & Female & $20(31.7)$ \\
\hline Age (years), median (range) & & $66(33-87)$ \\
\hline \multirow[t]{2}{*}{ Smoking History } & Yes & $36(57.1)$ \\
\hline & No & $27(42.9)$ \\
\hline Underlying COPD & & $3(4.8)$ \\
\hline \multirow[t]{5}{*}{ Tumor location ${ }^{a}$} & Right upper lobe & $15(23.8)$ \\
\hline & Right middle lobe & $6(9.5)$ \\
\hline & Right lower lobe & $20(31.7)$ \\
\hline & Left upper lobe & $19(30.2)$ \\
\hline & Left lower lobe & $14(22.2)$ \\
\hline \multirow[t]{6}{*}{ Histologic features } & Adenocarcinoma & $24(38.1)$ \\
\hline & Squamous cell carcinoma & $16(25.4)$ \\
\hline & Small cell lung cancer & $12(19.0)$ \\
\hline & Non-small cell lung cancer, NOS & $9(14.3)$ \\
\hline & Bronchoalveolar carcinoma & $1(1.6)$ \\
\hline & Large cell carcinoma & $1(1.6)$ \\
\hline \multirow[t]{4}{*}{ Aim of radiotherapy } & Definitive & $26(41.3)$ \\
\hline & Postoperative & $1(1.6)$ \\
\hline & Salvage & $19(30.1)$ \\
\hline & Palliative & $17(27.0)$ \\
\hline \multirow[t]{2}{*}{ Concurrent chemoradiotherapy } & Yes & $21(33.3)$ \\
\hline & No & $42(66.7)$ \\
\hline PTV (cc), median (range) & & $144(16-799)$ \\
\hline Total lung V10 (\%), median (range) & & $39(5-93)$ \\
\hline Total lung V20 (\%), median (range) & & $16(4-49)$ \\
\hline \multirow[t]{2}{*}{ Mode of tomotherapy } & Static & $12(19.0)$ \\
\hline & Helical & $51(81.0)$ \\
\hline
\end{tabular}


biopsy and staged according to the consensus recommendations of the International Association for the Study of Lung Cancer International Staging Committee [12].

All of the patients underwent helical tomotherapy $(N=51 ; 81.0 \%)$ or static tomotherapy $(N=12 ; 19.0 \%)$ for RT with definitive, salvage, or palliative treatment purposes. Tomotherapy was planned following the performance of chest computed tomography (CT), in which each patient was scanned from the mandible to the lower edge of the liver with 5-mm-thick slices. The gross tumor volume (GTV) included the primary tumor and involved regional lymphnodes, with a clinical target volume (CTV) of GTV plus a $0.6-0.8 \mathrm{~cm}$ margin and a planning target volume (PTV) of CTV plus a $0.5-1.0 \mathrm{~cm}$ margin. The patients usually received tomotherapy in five fractions per week at a radiation dose of 60-66 Gy in 2.0-2.2 Gy daily fractions.

Tomotherapy planning was performed using the TomoTherapy Hi-Art system version 4.0 (Accuray, Palo Alto, CA, USA). The planning system employed an inverse treatment planning process based on iterative least squares minimization of an objective function. The simultaneous integrated boost method was used in the planning for most patients. The importance factor placed constraints on the target and critical structures in relation to each other and determined the degree of weighting for achieving each prescription dose. For each target structure, a penalty factor was applied to the minimum and maximum dose values, and for organs at risk, it was used for the maximum dose. Each treatment plan was evaluated with a dose-volume histogram. In general, plans were considered acceptable if the PTV was covered by $95 \%$ of the isodose curves, inhomogeneity of the PTV ranged from $95 \%$ to $107 \%$, and doses to normal organs were limited in their tolerances. Dose tolerances for normal structures were as follows: total lung V $20<40 \%$, mean lung dose (MLD) $<20 \mathrm{~Gy}$, maximum dose to spinal cord $<45 \mathrm{~Gy}$, heart V60 $<33 \%$, and esophagus $\mathrm{V} 45<33 \%$.

Among the total of 63 patients, $21(33.3 \%)$ patients were treated with CCRT as either chemotherapy combined with static-mode tomotherapy (static-CCRT; $N=7$ ) or helical-mode tomotherapy (helical-CCRT; $N=14$ ). Chemotherapy was performed with a treatment regimen comprising cisplatin at a dose of $20-25 \mathrm{mg} / \mathrm{m}^{2} /$ day on days $1-3$ and docetaxel at $65-70 \mathrm{mg} / \mathrm{m}^{2} /$ day on day 1 , which was initiated simultaneously with tomotherapy. During the course of RT, 1-2 cycles of chemotherapy were concurrently administered, and after the complete of RT, additional 2-3 cycles of chemotherapy followed within 4 weeks.

The diagnosis of radiation pneumonitis was based on clinical symptoms, including dry cough, low-grade fever, shortness of breath, and chest pain, as well as the radiologic findings on chest X-ray and $\mathrm{CT}$ scan read by at least two radiologists. Most cases of acute radiation pneumonitis occur between 6 and 12 weeks after the conclusion of RT, whereas late radiation pneumonitis generally presents after 6 months [13]. The risk of radiation pneumonitis was evaluated by the grading systems of both the Radiation Therapy Oncology Group (RTOG) and the National Cancer Institute Common Terminology Criteria for Adverse Events (CTCAE), version 3.0 [14]. Our experimental protocols were approved by the Institutional Review Board of Severance Hospital at Yonsei University College of Medicine in Seoul, Korea.

\section{Animal model and treatment protocol}

Five-week-old Sprague-Dawley rats $(N=15$, body weights of $300-400 \mathrm{~g}$ ) were divided into five groups; Group 1: controls $(N=3)$, Group 2: rats treated with static tomotherapy alone $(N=3)$, Group 3: helical tomotherapy alone $(N=3)$, Group 4: static-CCRT $(N=3)$, and Group 5: helical-CCRT $(N=3)$. Chemotherapy was performed by intraperitoneal injection of gemcitabine $\left(2^{\prime}, 2^{\prime}\right.$-difluoro-2'deoxycytidine) (Eli Lilly and Company, Indianapolis, IN, USA) at a dose of $5 \mathrm{mg} / \mathrm{kg}$ body weight as described previously [15]. RT was planned by CT simulation and a dose of 12 Gy was delivered to the right lobe of the lung under anesthesia with phenobarbital at a dose of $30 \mathrm{mg} / \mathrm{kg}$ (Sigma-Aldrich Co. LLC, St. Louis, MO, USA). The left lung was protected by a constraint set to minimize radiation exposure in both helical and static tomotherapy. Target regions were irradiated with a margin of at least $2 \mathrm{~mm}$ in width from the center in order to prevent radiation esophagitis or myelitis. Other regions were protected from irradiation with a multileaf collimator. The animal studies were performed according to experimental protocols that were approved by the Committee for the Care and Use of Laboratory Animals of Severance Hospital. The experiments were repeated twice. Similar patterns were found between the first experiments and repeated experiments.

\section{Enzyme-linked immunosorbent assay}

Peripheral blood levels of IL-1 $\alpha$ (Abcam, Cambridge, UK) and IL-1 $\beta$ (Abcam) were detected using enzyme-linked immunosorbent assay (ELISA) kits according to the manufacturers' instructions. Peripheral blood samples were obtained from tail veins of experimental rats for 1,2 , and 3 weeks after tomotherapy and CCRT. For ELISA, $2 \mathrm{~mL}$ of peripheral blood was collected from each experimental rat followed by isolation of serum. Samples with inadequate serum quantity or quality due to hemolysis were excluded from biochemical measurements. Additionally, serum samples were measured in triplicate and those showing more than three-time variation from the mean value were excluded from the study. 


\section{Histological and immunohistochemical analysis}

Tissues from the right and left lungs of experimental animals were obtained at 3 weeks after tomotherapy or CCRT, fixed in $10 \%$ buffered formalin, and embedded in paraffin. Serial tissue sections of 4- $\mu \mathrm{m}$ thickness were prepared for histological analysis with hematoxylin and eosin (H\&E) stain. Additionally, immunohistochemical stains were performed with prepared tissue sections. Briefly, the sections were deparaffinized with xylene and then rehydrated in graded ethanol. Antigen retrieval was achieved by pressure-cooking using Antigen Retrieval Solution (Dako, Carpinteria, CA, USA). Then, endogenous peroxidase activity was blocked with Endogenous Enzyme Block (Dako). The sections were incubated with primary antibodies, including anti-IL-1 $\alpha$ antibody (Abcam) and rabbit anti-IL-1 $\beta$ polyclonal antibody (Santa Cruz Biotechnology, Santa Cruz, CA, USA) at a dilution of 1:100, rabbit antiIL-6 polyclonal antibody (Abcam) at 1:50, and mouse anti-TGF- $\beta$ monoclonal antibody (Abcam) at 1:25 for $1 \mathrm{~h}$ at room temperature. Real ${ }^{\mathrm{Tm}} \mathrm{EnVision}^{\mathrm{TN}}$ HRP Rabbit/Mouse detection system (Dako) was used as a secondary antibody, and the sections were then counterstained with hematoxylin.

Rabbit anti-IgG antibody (R\&D Systems GmbH, Wiesbaden, Germany) or mouse anti-IgG antibody (DakoCytomation $\mathrm{GmbH}$, Hamburg, Germany) were used as isotype-matched control antibodies at concentrations identical to those of the primary antibodies. The staining intensity of the tumor cells was scored as 0 (negative), 1 (light brown), 2 (brown), and 3 (dark brown). The final score was calculated as $(0 \times \%$ negative cells $)+(1 \times \%$ light brownstained cells $)+(2 \times \%$ brown-stained cells $)+(3 \times \%$ dark brown-stained cells) according to a weighted histoscore method [16].

\section{Statistical analysis}

Expression of IL- $1 \alpha$, IL-1 $\beta$, IL-6, and TGF- $\beta$ in each group was compared using the Mann-Whitney test. Statistical analyses were carried out using SPSS software (version 14.0; SPSS Inc., Chicago, IL, USA) and the differences were considered significant at a value of $p$ less than 0.05 .

\section{Results}

Frequency of pneumonitis in lung cancer patients treated with tomotherapy

The frequency of radiation pneumonitis was comparatively investigated in lung cancer patients who received tomotherapy using either the helical or the static mode. A total of 63 patients were evaluated for acute radiation pneumonitis, and 54 patients were then investigated for late radiation pneumonitis as 9 patients expired over the course of follow-up. The frequency of CCRT in the group of patients treated with helical-mode tomotherapy was higher than in those treated with the static mode $(p=0.04)$. However, both acute and late pneumonitis of $\geq$ grade 2 according to the RTOG and CTCAE scoring systems occurred more frequently in the group of patients treated with helical-mode tomotherapy than in those treated with the static mode over the course of RT (Table 2). Additionally, statistical analyses revealed that helical-mode tomotherapy resulted in a significantly higher rate of late radiation pneumonitis than static-mode tomotherapy when evaluated by the RTOG and CTCAE scoring system ( $p=0.001$, and 0.01 ).

Table 2 A comparison of patient characteristics and radiation pneumonitis in lung cancer patients treated with tomotherapy according to the delivery mode of radiation beams

\begin{tabular}{|c|c|c|c|c|}
\hline & & \multicolumn{2}{|c|}{ Mode of tomotherapy } & \multirow[t]{2}{*}{$P$-value } \\
\hline & & Static & Helical & \\
\hline \multirow[t]{2}{*}{ Smoking History } & Yes & $12 / 12(83.3 \%)$ & $26 / 51(51.0 \%)$ & \multirow[t]{2}{*}{0.04} \\
\hline & No & 2/12 (16.7\%) & $25 / 51(49.0 \%)$ & \\
\hline PTV (cc) & Median (range) & $158.50(57-799)$ & $144.00(16-789)$ & NS \\
\hline Total lung V10 (\%) & median (range) & $32.00(11-70)$ & $44.00(5-98)$ & NS \\
\hline Total lung V20 (\%) & median (range) & $16.00(5-51)$ & $16.00(4-53)$ & NS \\
\hline \multirow[t]{2}{*}{ CCRTX } & Yes & $7 / 12(58.3 \%)$ & 14/51 (27.5\%) & \multirow[t]{2}{*}{0.04} \\
\hline & No & $5 / 12(41.7 \%)$ & $37 / 51(72.5 \%)$ & \\
\hline \multicolumn{5}{|c|}{ Radiation pneumonitis scoring system } \\
\hline \multirow[t]{2}{*}{ RTOG } & Acute & $3 / 12(25.0 \%)$ & 15/51 (29.4 \%) & NS \\
\hline & Late & $1 / 12(8.3 \%)$ & $27 / 42(64.3 \%)$ & 0.001 \\
\hline \multirow[t]{2}{*}{ CTCAE } & Acute & $3 / 12(25.0 \%)$ & 20/51 (39.2\%) & NS \\
\hline & Late & $1 / 12(8.3 \%)$ & $20 / 42(47.6 \%)$ & 0.01 \\
\hline
\end{tabular}


Of the radiotherapeutic parameters, late grade 2 or worse radiation pneumonitis by CTCAE score was observed in $28.9 \%$ (11 of 38 patients) with a total lung V10 Gy of $<50 \%$ and in $62.5 \%$ (10 of 16 patients) with a total lung V20 Gy of $\geq 60 \%(p=0.02)$. Acute grade 2 or worse radiation pneumonitis by CTCAE scoring system was observed in $10(26.3 \%)$ of 38 patients with a total lung V20 Gy of $<20 \%$ and in $13(52.0 \%)$ of 25 patients with a total lung V20 Gy of $\geq 60 \%(p=0.04)$.

\section{Plasma levels of IL-1 $\alpha$ and IL-1 $\beta$ according to the experimental rat groups}

Plasma levels of IL- $1 \alpha$ and IL- $1 \beta$ in each experimental rat group were comparatively evaluated at 1 week, 2 weeks, and 3 weeks after tomotherapy or CCRT. No significant differences in the levels of expression of IL- $1 \alpha$ and IL-1 $\beta$ were found among the groups at 1 week or 2 weeks. However, there were significant differences among the groups at 3 weeks after tomotherapy or CCRT; expression of IL$1 \alpha$ was 1.8-fold (Group 2; $p=0.037$ ), 1.6-fold (Group 3 ; $p=0.037$ ), 3.1-fold (Group 4; $p=0.037$ ), and 3.2-fold (Group 5; $p=0.037$ ) higher compared to the control group (Group 1) (Fig. 1a), and expression of IL-1 $\beta$ was also 13.5fold (Group 2; $p=0.037$ ), 16.6-fold (Group 3; $p=0.037$ ), 20.3-fold (Group 4; $p=0.037$ ), and 27.1-fold (Group 5; $p=0.037$ ) higher (Fig. 1b).

The plasma levels of IL- $1 \alpha$ at 3 weeks after the treatments were significantly higher in the static-CCRT group (Group 4) compared to the groups that received either static tomography alone (Group 2; $p=0.047$ ) or helical tomotherapy alone (Group 3; $p=0.047$ ), as well as in the helical-CCRT group (Group 5) compared to the groups that received either static tomography alone $(p=0.047)$ or helical tomotherapy alone $(p=0.047)$. However, the plasma IL-1 $\alpha$ levels were not significantly different between the groups that received static tomotherapy alone and helical tomotherapy alone or between the staticCCRT group and the helical-CCRT group.
Additionally, the plasma levels of IL-1 $\beta$ at 3 weeks after the treatments were significantly higher in the static-CCRT group compared to the groups that received either static tomotherapy alone $(p=0.047)$ or helical tomography alone $(p=0.047)$, as well as in the helicalCCRT group compared to the groups that received either static tomotherapy alone $(p=0.047)$ or helical tomography alone $(p=0.047)$. Moreover, IL-1 $\beta$ expression was significantly increased in the helical tomotherapy alone group compared to the group that received static tomotherapy alone $(p=0.047)$, as well as in the helical-CCRT group compared to the static-CCRT group ( $p=0.047)$.

\section{Tissue expression of IL-1a in the animal model}

Lung specimens were obtained from the experimental rats in each group at 3 weeks after tomotherapy or CCRT, and tissue expression of IL- $1 \alpha$, IL- $1 \beta$, IL- 6 , and TGF- $\beta$ was evaluated using immunohistochemical staining and weighted histoscoring. Right lung samples from the treatment groups demonstrated 2.4-fold (Group 2, $p=0.037$ ), 2.8-fold (Group 3, $p=0.037$ ), 4.4-fold (Group 4, $p=0.037$ ), and 5.8-fold (Group 5, $p=0.037$ ) higher tissue expression of IL-1 $\alpha$ compared to the untreated right lung samples (Group 1) (Fig. 2a, c, d). Additionally, left lung samples from the treatment groups showed 1.5-fold (Group 2, $p=0.037$ ), 2.7-fold (Group 3, $p=0.037$ ), 4.2-fold (Group 4, $p=0.037$ ), and 5.4fold (Group 5, $p=0.037$ ) higher tissue expression of IL$1 \alpha$ compared to the untreated left lung specimens of Group 1 (Fig. 2b).

Both right and left lung samples obtained from the rats in groups treated with CCRT were found to have higher IL-1 $\alpha$ expression compared to the groups treated with RT alone (right lung, $p=0.004$; left lung, $p=0.004$ ). Additionally, rats treated with helical tomotherapy alone demonstrated significantly higher levels of IL-1 $\alpha$ compared to those treated with static tomotherapy alone (right lung, $p=0.047$; left lung, $p=0.047$ ). Helical-CCRT in rats resulted in significantly higher IL-1 $\alpha$ expression
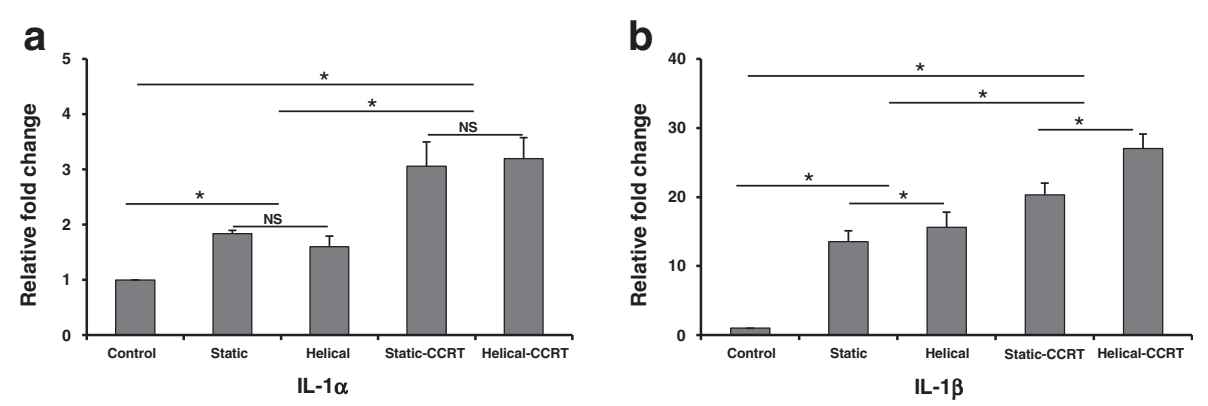

Fig. 1 Plasma levels of $\mathbf{a} \mid \mathrm{L}-1 \mathrm{a}$ and $\mathbf{b} \| \mathrm{L}-1 \beta$ according to the experimental rat groups. There were significant differences among the groups at 3 weeks after tomotherapy or CCRT. ${ }^{*} p<0.05$; NS, not significant; IL, interleukin; CCRT, concurrent chemoradiotherapy 

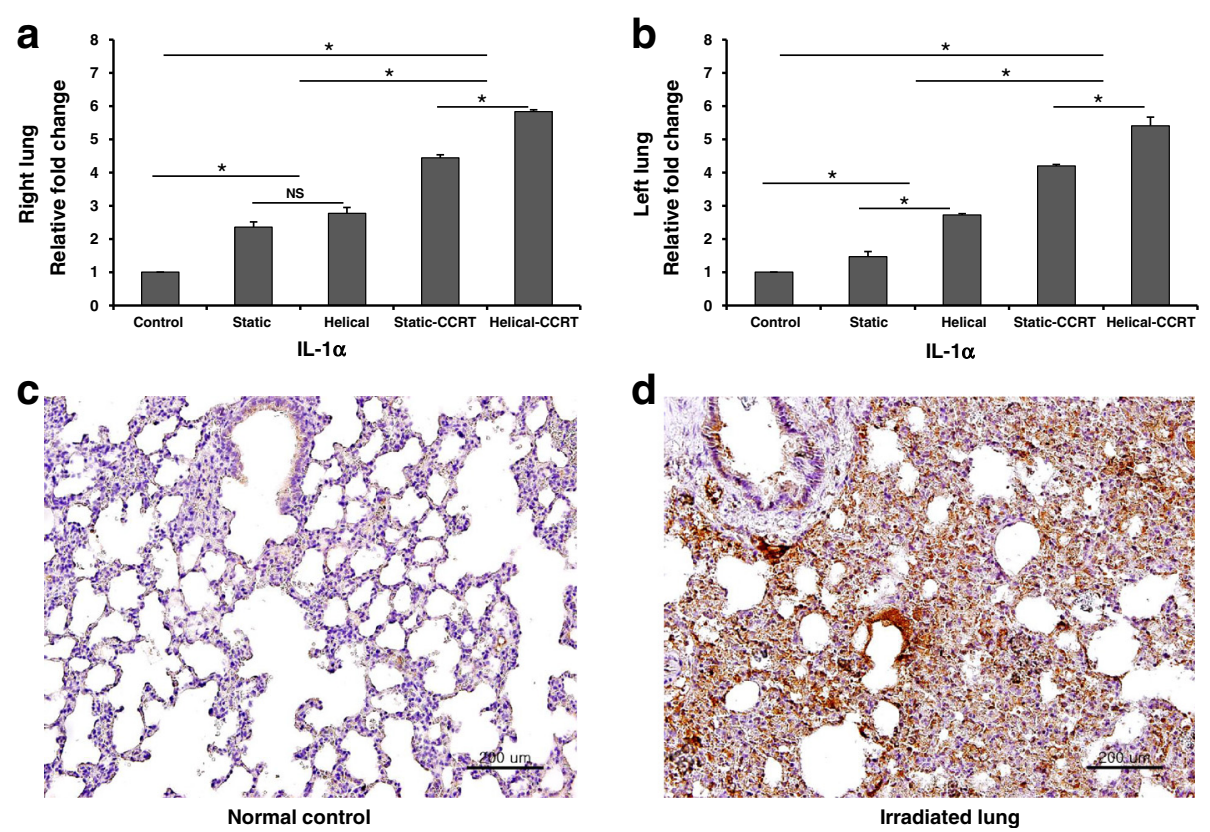

Fig. 2 Tissue expression of IL-1a. a Right lung samples demonstrated higher tissue IL-1a expression compared to the untreated control samples. b Left lung samples revealed upregulated tissue IL-1a expression. Immunohistochemical staining with anti-LL-1a antibody of $\mathbf{c}$ normal control and $\mathbf{d}$ right lung sample treated with helical tomotherapy with CCRT. ${ }^{*} p<0.05$; NS, not significant; IL, interleukin; CCRT, concurrent chemoradiotherapy; bars $=200 \mu \mathrm{m}$

compared to static-CCRT in both the right and left lungs ( $p=0.047$ and $p=0.047$, respectively).

\section{Tissue expression of IL-1 $\beta$ in the animal model}

Significantly upregulated tissue expression of IL-1 $\beta$ was found in both right and left lung samples obtained from rats in Group 2 (2.9-fold, $p=0.037$; 2.5 -fold, $p=0.037$, respectively), Group 3 (2.7-fold, $p=0.037 ; 2.9$ fold, $p=0.037$, respectively), Group 4 (3.7-fold, $p=0.037$; 3.3-fold, $p=0.037$, respectively), and Group 5 (3.8-fold, $p=0.037$; 3.7 -fold, $p=0.037$, respectively) when compared to the untreated control group (Group 1) (Fig. 3a-d). Both right and left lung samples obtained from the rats in groups treated with CCRT were found to have higher IL-1 $\beta$ expression compared to the groups treated with RT alone (right lung, $p=0.004$; left lung, $p=0.004$ ). Rats treated with helical tomotherapy alone demonstrated significantly higher tissue expression of IL-1 $\beta$ compared to those treated with static tomotherapy alone in the left lung $(p=0.047)$, but not in the right lung $(p>0.05)$. Additionally, rats treated with helical-CCRT showed significantly higher IL-1 $\beta$ levels compared to those treated with static-CCRT (right lung, $p>0.05$; left lung, $p=0.047$ ).

\section{Tissue expression of IL-6 in the animal model}

Significantly upregulated tissue expression of IL-6 was found in both right and left lung samples obtained from rats in Group 2 (5.2-fold, $p=0.037$; 3-fold, $p=0.037$, respectively), Group 3 (7.5-fold, $p=0.037$; 4-fold, $p=0.025$, respectively), Group 4 (10-fold, $p=0.034 ; 8.5$-fold, $p=0.037$, respectively), and Group 5 (14.5-fold, $p=0.037 ; 11$-fold, $p=0.037$, respectively) when compared to the untreated control group (Fig. 4a-d). Both right and left lung samples obtained from the CCRT-treated rats were found to have higher IL-6 levels compared to the groups treated with RT alone (right lung, $P=0.004$; left lung, $p=0.004)$. Rats treated with helical tomotherapy alone demonstrated significantly higher IL-6 expression compared to those treated with static tomotherapy alone (right lung, $p=0.047$; left lung, $p=0.037$ ). Additionally, helical-CCRT treatment resulted in remarkably higher tissue IL-6 expression compared to static-CCRT treatment (right lung, $p=0.046$; left lung, $p=0.047$ ).

\section{Tissue expression of TGF- $\beta$ in the animal model}

Right lung samples from the treatment groups demonstrated 15.7-fold (Group 2, $p=0.037$ ), 14.2-fold (Group 3, $p=0.037$ ), 20.3-fold (Group 4, $p=0.037$ ), and 25.3-fold (Group 5, $p=0.037$ ) higher tissue expression of TGF- $\beta$ compared to the right lung tissues of untreated controls (Fig. 5a, c, and d). Additionally, left lung samples from the treatment groups showed 2.5-fold (Group 2, $p=0.037$ ), 5.5fold (Group 3, $p=0.037$ ), 10.5-fold (Group 4, $p=0.037$ ), and 21.2-fold (Group 5, $p=0.037$ ) higher tissue TGF- $\beta$ expression compared to the controls (Fig. 5b). 

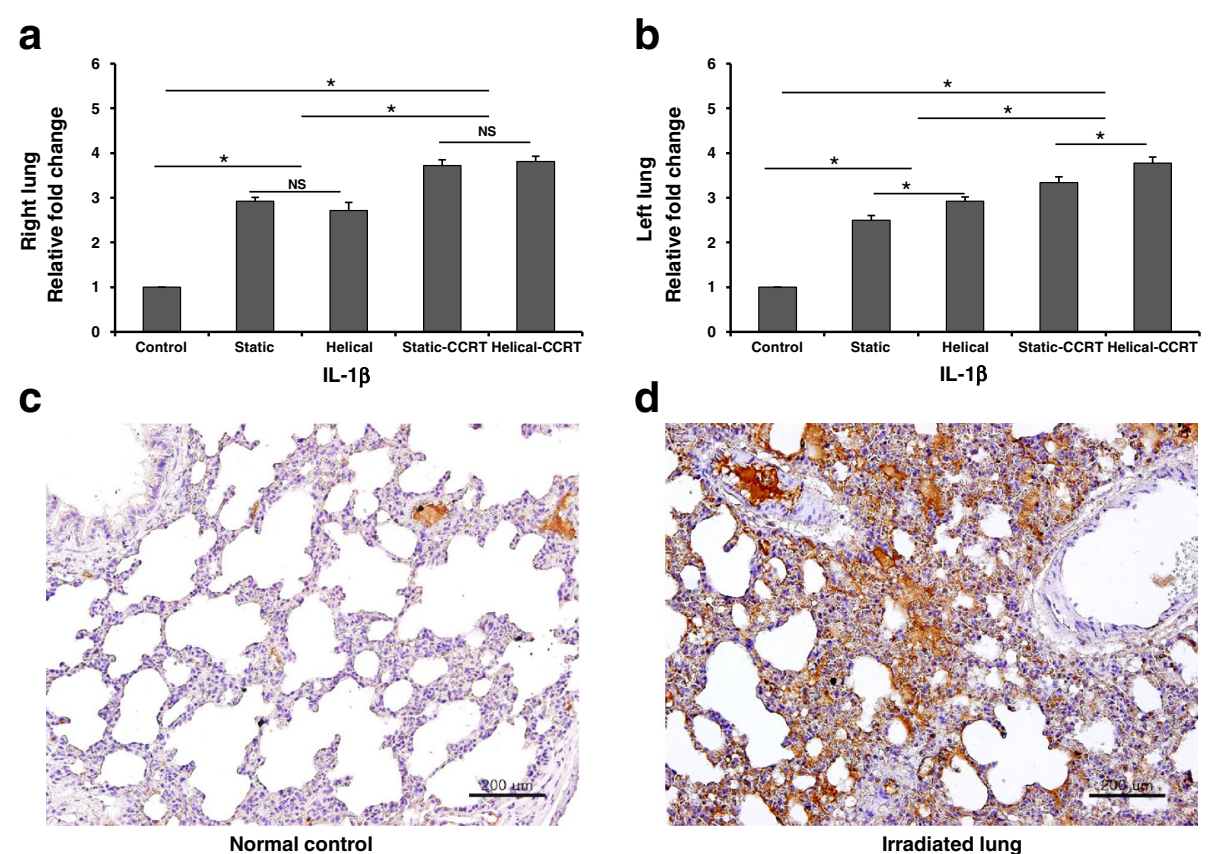

Fig. 3 Tissue expression of IL-1 3 . a Right lung samples demonstrated higher tissue IL-1ß expression compared to the untreated control samples. $\mathbf{b}$ Left lung samples revealed upregulated tissue IL-1 $\beta$ expression. Immunohistochemical staining with anti-IL-1 $\beta$ antibody of $\mathbf{c}$ normal control and $\mathbf{d}$ right lung sample treated with helical tomotherapy with CCRT. ${ }^{*} p<0.05$; NS, not significant; IL, interleukin; CCRT, concurrent chemoradiotherapy; bars $=200 \mu \mathrm{m}$
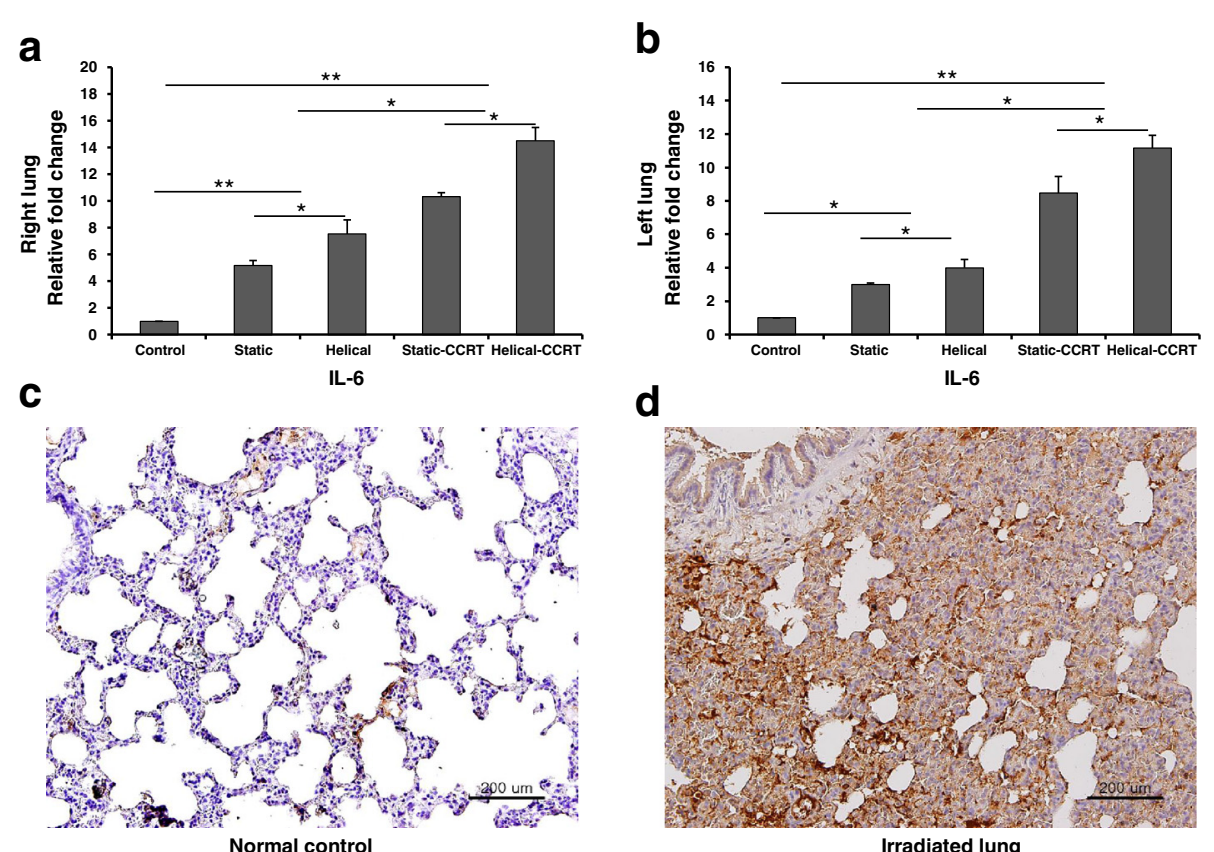

Fig. 4 Tissue expression of IL-6. a Right lung samples demonstrated higher tissue IL-6 expression compared to the untreated control samples. b Left lung samples revealed upregulated tissue IL-6 expression. Immunohistochemical staining with anti-IL-6 antibody of $\mathbf{c}$ normal control and $\mathbf{d}$ right lung sample treated with helical tomotherapy with CCRT. ${ }^{*} p<0.05 ;{ }^{* *} p<0.01$; IL, interleukin; CCRT, concurrent chemoradiotherapy; bars $=200 \mu \mathrm{m}$ 


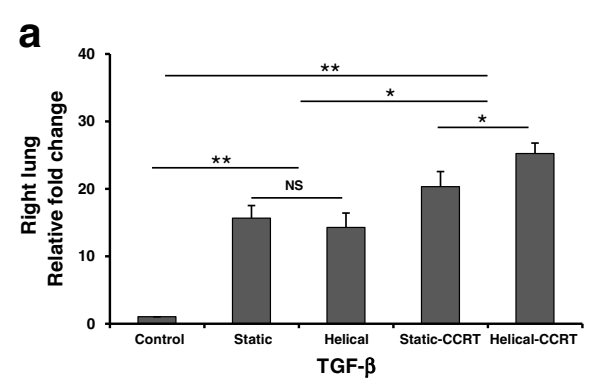

C

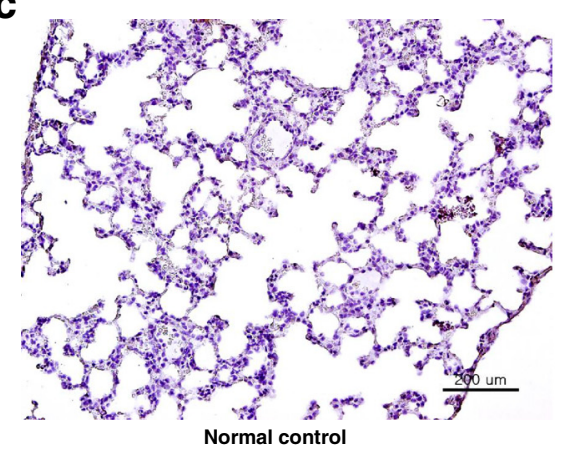

b

d
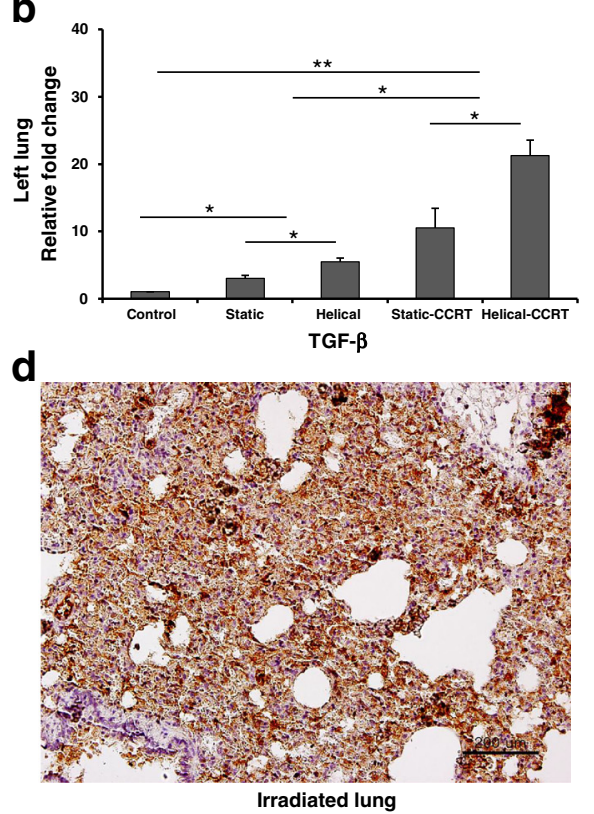

Fig. 5 Tissue expression of TGF- $\beta$. a Right lung samples demonstrated higher tissue TGF- $\beta$ expression compared to the untreated control samples. b Left lung samples revealed upregulated tissue TGF- $\beta$ expression. Immunohistochemical staining with anti-TGF- $\beta$ antibody of $\mathbf{c}$ normal control and $\mathbf{d}$ right lung sample treated with helical tomotherapy with CCRT. ${ }^{*} p<0.05 ;{ }^{* *} p<0.01$; NS, not significant; TGF, transforming growth factor; CCRT, concurrent chemoradiotherapy; bars $=200 \mu \mathrm{m}$

Both right and left lung samples obtained from the rats in groups treated with CCRT were found to have remarkably higher TGF- $\beta$ expression compared to the RT alone groups (right lung, $p=0.006$; left lung, $p=0.004$ ). Helical tomotherapy alone induced significantly higher TGF- $\beta$ expression in lung specimens compared to static tomotherapy alone (right lung, $p>0.05$; left lung, $p=0.047$ ). Additionally, rats treated with helical-CCRT demonstrated significantly higher TGF- $\beta$ expression compared to those treated with static-CCRT (right lung, $p=0.047$; left lung, $p=0.047$ ).

\section{Discussion}

Radiation-induced pneumonitis occurs in various cancerous conditions, including small cell or non-small cell carcinoma of the lung, esophageal cancer, breast cancer, and malignant lymphoma, as a complication of thoracic RT [17]. In patients with lung cancers, the risk of radiation pneumonitis is significantly correlated with the dose and volume factors of RT [18]. When the total lung volume receiving $20 \mathrm{~Gy}$ is less than $22 \%$, radiation pneumonitis rarely occurs, whereas an $8 \%$ risk of grade 2 radiation pneumonitis can be encountered in cases with $22-31 \%$ of the lung volume receiving 20 Gy [18]. Additionally, a dose-volume histogram analysis study demonstrated that the dosimetric factors of normal tissue complication probability, mean dose, and total lung volume receiving $\geq 30$ Gy were significantly associated with radiation-induced pneumonitis [19].
The lung volume receiving high doses has been reduced by the advent of IMRT, however, the risk of acute or late pulmonary toxicity should be considered as the volume receiving low doses has been increased $[17,20,21]$. Helical-mode tomotherapy delivers low-dose exposure of almost the entire lung volume at target area level. Several authors reported helical tomotherapy is safe and not associated with a specific pattern of lung injury [22]. Donato et al. assessed the toxicities of hypofractionated radiation course delivered with helical tomotherapy, and they reported acute grade 3 treatment-related pneumonitis was detected in $10 \%$ of patients. These findings not increased the risk of radiation pneumonitis when compared with other observed studies.

Song et al. assessed clinical outcomes in non-smallcell lung cancer patients treated with chemotherapy and helical tomotherapy, and reported four patients died of treatment-related death after helical-mode radiotherapy [23]. They suggested that helical tomotherapy has high rate of fatal pulmonary complications. Kim et al. reported low dose V5 of ipsilateral and contralateral lung volume were also significant predictive factors for radiation pneumonitis after helical tomotherapy [24]. They recommended normal lung of V5, V10 and V15 should be kept as low as possible. To reduce the chance of lowdose irradiation to large areas of normal tissue, a type of static tomotherapy, which uses static gantry positions, has been performed in combination with multileaf 
collimator modulation and couch translation [11, 25]. The planning time of IMRT can be reduced in staticmode tomotherapy by using specified beam angles compared with the helical-mode [11, 25]. Additionally, the beam-on time is lesser in the static-mode than in the helical mode and conventional 3-dimensional conformal RT [11]. Therefore, optimal target volume coverage with adequate sparing of normal tissue (e.g.,lung volume) can be achieved by using static-mode tomotherapy [25].

In this study, we compared the risk of radiation pneumonitis between helical tomotherapy and static tomotherapy in lung cancer patients. Although, the frequency of CCRTx in the group of patients treated with helical-mode tomotherapy was higher than in those treated with the static mode $(p=0.04)$, they found that helical-mode tomotherapy resulted in a significantly higher rate of late radiation pneumonitis than static-mode tomotherapy.

Additionally, we developed rat models of radiation pneumonitis, which were induced by helical tomography and static tomography. Our evaluation of tissue samples from treated lung found that the expression of radiationinduced inflammatory cytokines, including TGF- $\beta, \mathrm{IL}-1 \alpha$, IL-1 $\beta$, and IL-6, was similarly increased in groups treated with helical-mode and static-mode tomotherapy. However, when comparing samples from the opposite side of the lung, we found that radiation-induced inflammatory cytokines were significantly increased in rat groups treated with helical-mode tomotherapy compared to those treated with static-mode tomotherapy.

The risk of radiation-induced pulmonary toxicity is remarkably increased by the concomitant systemic therapy of CCRT [17]. However, dose-volume recommendations which are specific for CCRT have not been fully established, with the exception of the recommendations of total lung volume receiving 20 Gy and 30 Gy [17-19]. In our study, we found that animal models also exhibited significantly remarkable pulmonary toxicity on the treated side of the lungs in the groups that received CCRT compared to those treated with helical-mode and static-mode tomotherapy alone. Moreover, when comparing the opposite side of the lung, we found that radiationinduced inflammatory cytokines were increased in the rat group treated with helical-CCRT compared to the staticCCRT group.

\section{Conclusion}

In the present study, we found that rat models treated with helical-mode or static-mode tomotherapy with or without CCRT could present similar patterns of pulmonary toxicity to those shown in lung cancer patients. Nevertheless, further studies are needed to establish an appropriate animal model of radiation-induced pulmonary toxicity to exactly reflect the radiation-induced human tissue response. Additionally, we suggest that our study can be used as a basic reference for developing an animal model of radiation-induced pulmonary toxicity.

\section{Abbreviations}

RT: Radiotherapy; IMRT: Intensity-modulated radiotherapy; CCRT: Concurrent chemoradiotherapy; RTOG: Radiation Therapy Oncology Group; CTCAE: Cancer Institute Common Terminology Criteria for Adverse Events; IL: Interleukin; TGF: Transforming growth factor; CT: Computed tomography; GTV: Gross tumor volume; CTV: Clinical target volume; PTV: Planning target volume; MLD: Mean lung dose; ELISA: Enzyme-linked immunosorbent assay; H\&E: Hematoxylin and eosin.

\section{Competing interests}

The authors declare that they have no competing interests.

\section{Authors' contributions}

YKS and ZLZh carried out the in vivo studies. LHZ carried out data collection, and analysis. XLZ and IJL participated in the design of the study and drafted the manuscript. All authors read and approved the final manuscript.

\section{Acknowledgements}

This work was supported by the National Research Foundation of Korea (NRF) grant funded by the Korean government (MEST) (No. 2010-0004085) and a faculty research grant of Yonsei University College of Medicine for 2015 (Grant No. 6-2015-0009).

\section{Author details}

'Department of Pathology, Yanbian University Hospital, Yanji, China. ${ }^{2}$ Oral Cancer Research Institute, Yonsei University College of Dentistry, Seoul, South Korea. ${ }^{3}$ Cancer Metastasis Research Center, Yonsei University College of Medicine, Seoul, South Korea. ${ }^{4}$ Department of Dermatology, Yanbian University Hospital, Yanji, China. 'Department of Radiation Oncology, Gangnam Severance Hospital, Yonsei University College of Medicine, 211 Eonju-ro, Gangnam-gu, Seoul 135-720, South Korea.

Received: 20 June 2015 Accepted: 4 September 2015

Published online: 17 September 2015

\section{References}

1. Vogelius IR, Westerly DC, Aznar MC, Cannon GM, Korreman SS, Mackie TR, et al. Estimated radiation pneumonitis risk after photon versus proton therapy alone or combined with chemotherapy for lung cancer. Acta Oncol. 2011;50:772-6.

2. Baskar R, Lee KA, Yeo R, Yeoh KW. Cancer and radiation therapy: current advances and future directions. Int J Med Sci. 2012;9:193-9.

3. Reynders T, Tournel K, De Coninck P, Heymann S, Vinh-Hung V, Van Parijs H, et al. Dosimetric assessment of static and helical TomoTherapy in the clinical implementation of breast cancer treatments. Radiother Oncol. 2009;93:71-9.

4. Song C, Pyo H, Kim J, Lim YK, Kim WC, Kim HJ, et al. Superiority of conventional intensity-modulated radiotherapy over helical tomotherapy in locally advanced non-small cell lung cancer. A comparative plan analysis. Strahlenther Onkol. 2012;188:901-9.

5. Zibold F, Sterzing F, Sroka-Perez G, Schubert K, Wagenknecht K, Major G, et al. Surface dose in the treatment of breast cancer with helical tomotherapy. Strahlenther Onkol. 2009;185:574-81.

6. Katayama S, Hantschke M, Lissner S, Lindel K, Oetzel D, Herfarth K, et al. Helical tomotherapy of the complete scalp and the ipsilateral lymph nodes in a case of scalp angiosarcoma. Ear Nose Throat J. 2014;93:E24-8.

7. Acevedo-Henao CM, Lopez Guerra JL, Matute R, Puebla F, Russo M, Rivin E, et al. Image-guided radiation therapy based on helical tomotherapy in prostate cancer: minimizing toxicity. Oncol Res Treat. 2014;37:324-30.

8. Kong M, Hong SE, Choi WS, Choi J, Kim Y. Treatment outcomes of helical intensity-modulated radiotherapy for unresectable hepatocellular carcinoma. Gut Liver. 2013;7:343-51.

9. Nagai A, Shibamoto Y, Yoshida M, Inoda K, Kikuchi Y. Safety and efficacy of intensity-modulated stereotactic body radiotherapy using helical tomotherapy for lung cancer and lung metastasis. Biomed Res Int. 2014;2014:473173.

10. Wiezorek T, Brachwitz T, Georg D, Blank E, Fotina I, Habl G, et al. Rotational IMRT techniques compared to fixed gantry IMRT and tomotherapy: multiinstitutional planning study for head-and-neck cases. Radiat Oncol. 2011;6:20. 
11. Borca VC, Franco P, Catuzzo P, Migliaccio F, Zenone F, Aimonetto S, et al. Does TomoDirect 3DCRT represent a suitable option for post-operative whole breast irradiation? A hypothesis-generating pilot study. Radiat Oncol. 2012;7:211.

12. Goldstraw P, Crowley J, Chansky K, Giroux DJ, Groome PA, Rami-Porta R, et al. The IASLC Lung Cancer Staging Project: proposals for the revision of the TNM stage groupings in the forthcoming (seventh) edition of the TNM Classification of malignant tumours. J Thorac Oncol. 2007;2:706-14.

13. Movsas B, Raffin TA, Epstein AH, Link Jr CJ. Pulmonary radiation injury. Chest. 1997;111:1061-76.

14. Tucker SL, Jin H, Wei X, Wang S, Martel MK, Komaki R, et al. Impact of toxicity grade and scoring system on the relationship between mean lung dose and risk of radiation pneumonitis in a large cohort of patients with non-small cell lung cancer. Int J Radiat Oncol Biol Phys. 2010;77:691-8.

15. Mason KA, Milas L, Hunter NR, Elshaikh M, Buchmiller L, Kishi K, et al. Maximizing therapeutic gain with gemcitabine and fractionated radiation. Int J Radiat Oncol Biol Phys. 1999;44:1125-35.

16. Witton CJ, Hawe SJ, Cooke TG, Bartlett JM. Cyclooxygenase 2 (COX2) expression is associated with poor outcome in ER-negative, but not ER-positive, breast cancer. Histopathology. 2004;45:47-54

17. Chargari C, Riet F, Mazevet M, Morel E, Lepechoux C, Deutsch E. Complications of thoracic radiotherapy. Presse Med. 2013;42:e342-51.

18. Graham MV, Purdy JA, Emami B, Harms W, Bosch W, Lockett MA, et al. Clinical dose-volume histogram analysis for pneumonitis after 3D treatment for non-small cell lung cancer (NSCLC). Int J Radiat Oncol Biol Phys. 1999:45:323-9.

19. Hernando ML, Marks LB, Bentel GC, Zhou SM, Hollis D, Das SK, et al. Radiation-induced pulmonary toxicity: a dose-volume histogram analysis in 201 patients with lung cancer. Int J Radiat Oncol Biol Phys. 2001;51:650-9.

20. Murshed H, Liu HH, Liao Z, Barker JL, Wang X, Tucker SL, et al. Dose and volume reduction for normal lung using intensity-modulated radiotherapy for advanced-stage non-small-cell lung cancer. Int J Radiat Oncol Biol Phys. 2004:58:1258-67.

21. Liu HH, Wang X, Dong L, Wu Q, Liao Z, Stevens CW, et al. Feasibility of sparing lung and other thoracic structures with intensity-modulated radiotherapy for non-small-cell lung cancer. Int J Radiat Oncol Biol Phys. 2004;58:1268-79.

22. Arcangeli S, Agolli L, Portalone L, Migliorino MR, Lopergolo MG, Monaco A, et al. Patterns of $C T$ lung injury and toxicity after stereotactic radiotherapy delivered with helical tomotherapy in early stage medically inoperable NSCLC. Br J Radiol. 2015:88:20140728.

23. Song $\mathrm{CH}$, Pyo H, Moon SH, Kim TH, Kim DW, Cho KH. Treatment-related pneumonitis and acute esophagitis in non-small-cell lung cancer patients treated with chemotherapy and helical tomotherapy. Int J Radiat Oncol Biol Phys. 2010;78:651-8.

24. Kim $\mathrm{Y}$, Hong $\mathrm{SE}$, Kong $\mathrm{M}$, Choi J. Predictive factors for radiation pneumonitis in lung cancer treated with helical tomotherapy. Cancer Res Treat. 2013;45:295-302.

25. Lee HC, Kim SH, Suh YJ, Chung MJ, Kang DG, Choi HJ, et al. A prospective cohort study on postoperative radiotherapy with TomoDirect using simultaneous integrated boost technique in early breast cancer. Radiat Oncol. 2014;9:244.

\section{Submit your next manuscript to BioMed Central and take full advantage of:}

- Convenient online submission

- Thorough peer review

- No space constraints or color figure charges

- Immediate publication on acceptance

- Inclusion in PubMed, CAS, Scopus and Google Scholar

- Research which is freely available for redistribution 